\title{
THz Metal Mesh filters on Electrically Thick Fused Silica Substrates
}

\author{
W. J. Otter, F. Hu, J. Hazell and S. Lucyszyn \\ Centre for Terahertz Science and Engineering, Imperial College London \\ Optical and Semiconductor Devices Group, Electrical and Electronic Engineering, Imperial College London
}

\begin{abstract}
This paper shows simulated and measured results of ultra-low cost metal mesh filters on electrically thick substrates for millimeter-wave and $\mathrm{THz}$ bands. It provides a broad overview of metal mesh filters currently available and suggest why it is worth moving to an electrically thick substrate for ultralow cost applications. We demonstrate scalable traditional metal mesh filters on $525 \mu \mathrm{m}$ thick fused silica substrates. In addition, trapped-mode excitation is exploited to improve out-of-band rejection at higher frequencies. The measured results prove that these filters are scalable in the $\mathrm{THz}$ range using cost-effective micromachining manufacturing. This work opens up the possibility of using electrically thick metal mesh filters for ultralow cost applications.
\end{abstract}

\section{INTRODUCTION}

$\mathrm{M}$ ETAMATERIALS based terahertz devices (e.g. sensors and filters), amplitude/phase and spatial modulators provide methods to control and manipulate $\mathrm{THz}$ waves [1]. One simple example is metal mesh filters [2-3], which have been widely used in applications such as radio astronomy and are commercially available from 0.1 to $30 \mathrm{THz}$. A fundamental limitation in self-supporting film designs is that at higher frequencies the metal becomes electrically thick, thus unwanted waveguide modes can be excited within the structure [4]. Thin metallic films have been demonstrated on electrically thin substrates or encased within polymer layers to reduce the waveguide effect [5]. However, thin substrates are fragile and expensive and encasing the film within a polymer increases the cost due to the extra fabrication steps.

In our work, we demonstrate experimentally that, with careful design, standard fused silica wafers that are electrically thick can be used for low cost, high performance and frequency-scalable ultra-thin metal mesh filters.

\section{DESIGN AND FABRICATION}

Two metal mesh filter designs are considered; the traditional cross-shaped and improved trapped-mode [6] designs. The initial dimensions of the filters were calculated using standard techniques for thin substrate [2]. These were then adapted for electrically thick substrates, by a careful choice of substrate material, thickness and further optimization. A $525 \mu \mathrm{m}$ thick fused silica substrate was selected, as it has low loss at THz frequencies, a low dielectric constant and is compatible with traditional surface micromachining.

The main challenge with the filter design is the relatively thick substrate. Firstly the use of thick substrates detunes the filter towards a longer wavelength. This wavelength shift is dependent on the refractive index of the substrate, $n_{2}$, and is fully described by:

$$
\lambda_{R} \rightarrow \lambda_{R 0} \sqrt{\frac{\left(n_{1}^{2}+n_{2}^{2}\right)}{2}}
$$

where $\lambda_{R}$ is the center frequency of the filter with substrate added, $\lambda_{R 0}$ is the filter's center wavelength in free-space and $n_{1}$ is the refractive index of the materials on the non-substrate side of the mesh, nominally free space (with $n=1$ ).

Furthermore, if a high permittivity substrate is chosen, a Fabry-Perot elaton is created, which reduces the out-of-band rejection and causes interference with the mesh filter response. A method of improving the out-of-band rejection is to add an inner cross, producing a trapped-mode excitation [6], which has the opposite surface current to the outer structure as shown in Fig. 1. Therefore, only waves at or near the resonance frequency will be able to pass through this structure.

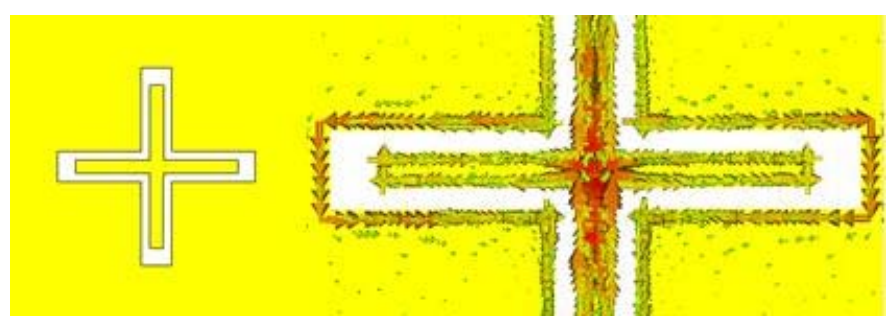

Fig. 1: (left) Trapped-mode filter shape and (right) simulated current distribution in the metal.

The simulated results for a conventional $0.4 \mathrm{THz}$ crossshaped and a $0.3 \mathrm{THz}$ trapped-mode metal mesh filters are shown in Fig. 2. The trapped-mode design shows better outof-band rejection and higher Q-factor when compared to the conventional cross-shaped filters.

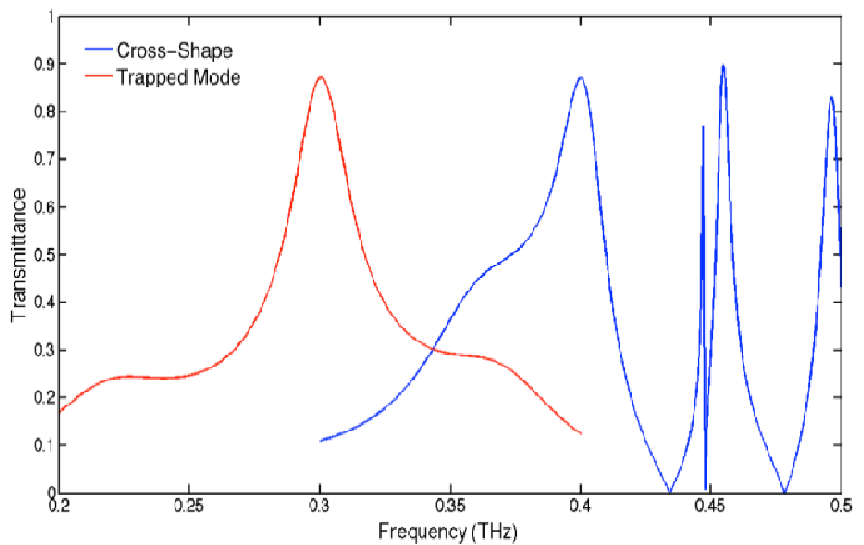

Fig. 2: Simulated results for (a) conventional $0.4 \mathrm{THz}$ cross-shaped metal mesh filter and (b) $0.3 \mathrm{THz}$ trapped-mode metal mesh filter.

The filters were fabricated on a $100 \mathrm{~mm}$ diameter fused silica substrate. Fig. 3 shows the fabrication steps; initially the metallization layer consisting of a $35 \mathrm{~nm}$ thick chrome seed layer and a $150 \mathrm{~nm}$ thick gold layer, which are sputter coated on the substrate. The metallization layer was chosen to be ultrathin, in order to avoid waveguide modes within the crosses. A layer of photoresist was then spun on the wafer and, using standard photolithographic techniques and a low cost acetate mask, it was exposed and developed. When fully developed, the gold and chrome layers were wet etched; the result of this wet etch are shown in Fig. 4. The photoresist was 
then removed and the structure cleaned with acetone and IPA. Finally, the wafer was diced to separate the filters for measurement.

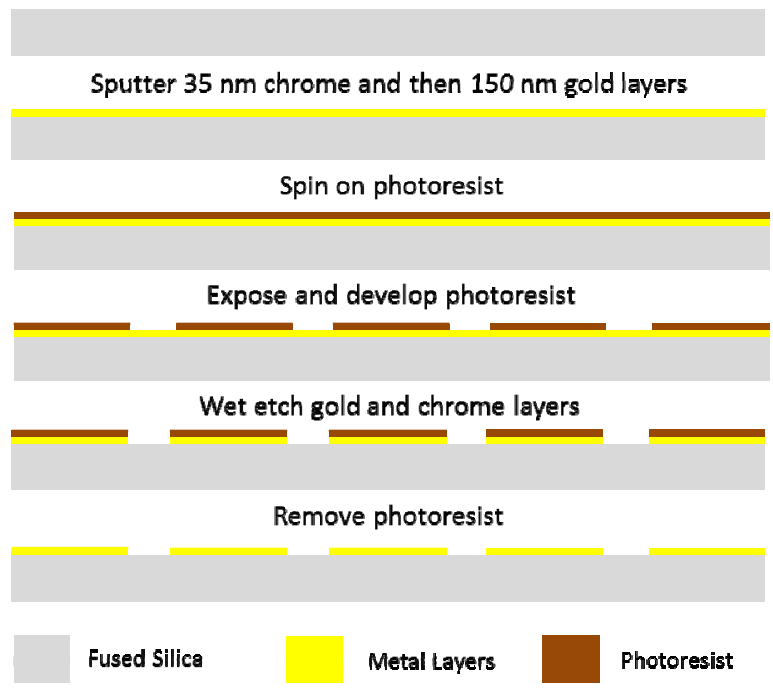

Fig. 3: Microfabrication processing steps for the filters.

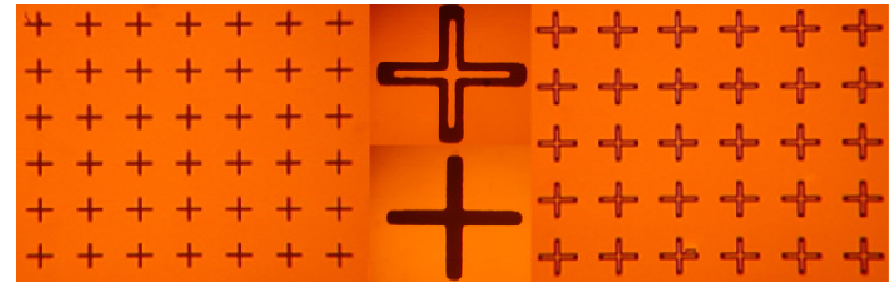

Fig. 4: Microscope images of both (left) cross-shaped and (right) trappedmode metal mesh THz filters. The centre inserts show individual (bottom) cross-shaped and (top) trapped-mode crosses.

\section{MEASURED RESULTS}

The filters were measured using our turnkey TeraView Spectra 3000 terahertz time-domain spectroscopy (THz-TDS) system. The broadband measurements from 0.06 to $3 \mathrm{THz}$ are as shown in Fig. 5. As expected, the conventional $0.4 \mathrm{THz}$ cross-shaped filter shows a pass band at $0.4 \mathrm{THz}$; however, a second mode at $0.5 \mathrm{THz}$ is also observed. The improved 0.3 THz trapped-mode filter shows better out-of-band rejection, as well as an increase in pass band transmittance.

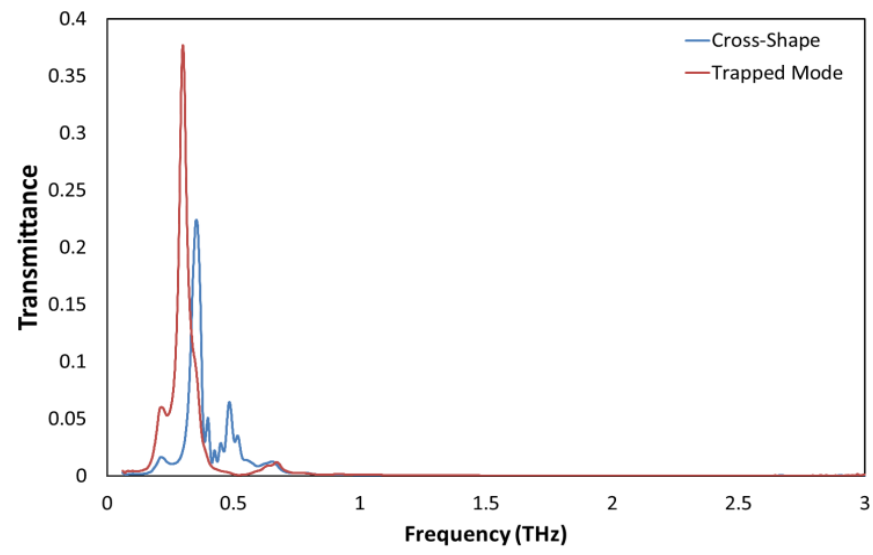

Fig. 5: Measurements in an $N_{2}$ purged chamber for the transmittance of 0.4 $\mathrm{THz}$ cross-shaped and $0.3 \mathrm{THz}$ trapped-mode metal mesh filters.

\section{SUMMARY}

We have demonstrated the design, fabrication and measurements for the implementation of conventional crossshaped and improved trapped-mode scalable ultra-thin metallic film $\mathrm{THz}$ filters on electrically thick substrates. This work proves that it is possible to realize easy-to-manufacture and high performance metal mesh filters on thick substrates for low-cost millimeter-wave and $\mathrm{THz}$ applications.

\section{REFERENCES}

[1] S. Lucyszyn, F. Hu and W. J. Otter, "Technology demonstrators for lowcost terahertz engineering", 2013 Asia-Pacific Microwave Conf. (APMC2013), Seoul, South Korea, Nov. 2013 (Invited)

[2] A. M. Melo, A. L. Gobbi, M. H. O. Piazzetta, and A M. P. A. da Silva "Cross-shaped terahertz metal mesh filters: Historical review and results", Adv. Opt. Tech., Jan. 2012

[3] O. Sternberg, "Resonances of periodic metal-dielectric meshes in the infrared wavelength region", Ph.D. Dissertation, New Jersey Institute of Technology, 2002

[4] Y. Wang, B. Yang, Y. Tian, R. S. Donnan, M. J. Lancaster, "Micromachined thick mesh filters for millimeter-wave and terahertz applications," IEEE Trans. Terahertz Sci. Technol., vol.4, no.2, pp. 247-253, Mar. 2014

[5] F. Pavanello, F. Garet, M-B. Kuppam, E. Peytavit, M. Vanwolleghem, F. Vaurette, J-L. Coutaz and J-F. Lampin, "Broadband ultra-low-loss mesh filters on flexible cyclic olefin copolymer films for terahertz applications" Appl. Phy. Lett., vol. 102, Mar. 2013

[6] O. Paul, R. Beigang, and M. Rahm, "Highly selective terahertz bandpass filters based on trapped mode excitation", Opt. Express, vol. 17, no. 21, pp. 18590-18595, Oct. 2009 